\title{
The phi-ratio tests
}

\author{
Sayel Ali and Marion Deutsche Cohen
}

Sayel Ali received his B.Sc. in Mathematics from the University of Jordan, M.Sc. in Mathematics from the University of Dundee (Scotland), and Ph.D. in Mathematics from the Ohio State University, USA. He teaches at the department of Mathematics at Minnesota State University Moorhead in Moorhead, Minnesota, USA.

Marion Deutsche Cohen received her Ph.D. from Wesleyan University in Connecticut, USA. Her thesis was on a theorem in Schwartz distribution theory, with generalizations in abstract algebra, and her advisor "in absentia" was Laurent Schwartz. She teaches at Arcadia University in Glenside, Pennsylvania. She is also a poet, writer, and memoirist.

In [1], a family of new convergence tests, the $m$-th ratio tests, was established. These tests are stronger than the ordinary ratio test; that is, they succeed in testing many series for which the ordinary ratio test fails.

The $m$-th ratio test says that the convergence of the series $\sum_{n=1}^{\infty} a_{n}$ depends upon the values of the lim inf and the lim sup of the $m$ ratios $\frac{a_{m n}}{a_{n}}, \frac{a_{m n+1}}{a_{n}}, \ldots, \frac{a_{m n+m-1}}{a_{n}}$ as $n \rightarrow \infty$. In the special case of series with positive decreasing terms, convergence depends only upon the value of $\lim _{n \rightarrow \infty} \frac{a_{m n}}{a_{n}}$. The series converges if $\lim _{n \rightarrow \infty} \frac{a_{m n}}{a_{n}}<\frac{1}{m}$, and diverges if $\lim _{n \rightarrow \infty} \frac{a_{m n}}{a_{n}}>\frac{1}{m}$. In this paper we will generalize the case of the $m$-th ratio test that applies to series with positive decreasing terms. We will replace the ratio $\frac{a_{m n}}{a_{n}}$ with the ratio $\frac{a_{\varphi(n)}}{a_{n}}$, where $\varphi$ : $Z^{+} \rightarrow Z^{+}$satisfies $0<\lim _{n \rightarrow \infty} \frac{n}{\varphi(n)}<1$. For any $m \in Z^{+}$, the case $\varphi(n)=m n$ is included in [1]. For this reason our new test is called, for any such $\varphi$, the $\varphi$-ratio test. According to the main result of this paper, the convergence or divergence of a series $\sum_{n=1}^{\infty} a_{n}$ with

Tests für die Konvergenz oder Divergenz von Reihen gehören zum Grundinventar jeder Einführungsvorlesung in Analysis. Quotientenkriterium, Wurzelkriterium, Vergleichskriterium, Integralkriterium, das Cauchy-Verdichtungskriterium oder das Leibniz-Kriterium sind bekanntere Varianten. Daneben gibt es zahlreiche weitere Konvergenztests, etwa die Kriterien von Dirichlet, Abel, Raabe oder Kummer. Von Augustus De Morgan stammt eine Hierarchie, welche diese Tests nach ihrer Schärfe klassifiziert. Die Autoren des vorliegenden Artikels untersuchen Reihen mit positiven, monoton fallenden Gliedern und verallgemeinern eine Variante des Quotiententests. 
positive decreasing terms depends upon the value of $\lim _{n \rightarrow \infty} \frac{a_{\varphi(n)}}{n}$, as long as that value is not equal to $\lim _{n \rightarrow \infty} \frac{n}{\varphi(n)}$.

In what follows, for $\varphi: Z^{+} \rightarrow Z^{+}$and for $k \in Z^{+}, \varphi^{k}$ will denote the $k$-th iterate of $\varphi$. Thus $\varphi^{0}$ will denote the identity on $Z^{+}$.

Lemma. Let $\varphi: Z^{+} \rightarrow Z^{+}$be such that $\lim _{n \rightarrow \infty} \frac{n}{\varphi(n)}$ exists. Denote $\lim _{n \rightarrow \infty} \frac{n}{\varphi(n)}$ by $\alpha$ and suppose $0<\alpha<1$. Then

(A) There is an $N \in Z^{+}$such that, for any $n \geq N$, $\left\{\varphi^{k}(n)\right\}$ is a strictly increasing subsequence (in $k$ ) of $Z^{+}$.

(B) For such $N, \lim _{k \rightarrow \infty} \frac{\varphi^{k+1}(N)-\varphi^{k}(N)}{\varphi^{k}(N)-\varphi^{k-1}(N)}=\frac{1}{\alpha}$.

Proof. Since $0<\alpha<1$ and $\lim _{n \rightarrow \infty} \frac{n}{\varphi(n)}=\alpha$, we have $\lim _{n \rightarrow \infty} \frac{\varphi(n)}{n}>1$. Therefore there exists $N \in Z^{+}$such that $\frac{\varphi(n)}{n}>1$ for all $n \geq N$ and therefore $\varphi(n)>n$ and $\varphi(n)>N$ for all $n \geq N$. This inequality implies the following two inequalities:

(i) For any $n \geq N$ and any $k \in Z^{+}, \varphi^{k}(n)>n$.

(ii) For any $n \geq N$ and any $k \in Z^{+}, \varphi^{k}(n)>\varphi^{k-1}(n)$.

Inequality (i) follows by induction on $k$. Inequality (ii) follows from (i) by replacing $n$, in $\varphi(n)>n$, with $\varphi^{k-1}(n)$. This is permissible since, from (i), $\varphi^{k-1}(n) \geq n \geq N$. Clearly, (ii) proves (A).

To prove (B), we observe that (A) implies $\lim _{k \rightarrow \infty} \varphi^{k}(n)=\infty$ for any $n \geq N$, and thus $\lim _{k \rightarrow \infty} \varphi^{k-1}(N)=\infty$. Therefore,

and

$$
\lim _{k \rightarrow \infty} \frac{\varphi^{k+1}(N)}{\varphi^{k}(N)}=\lim _{k \rightarrow \infty} \frac{\varphi\left(\varphi^{k}(N)\right)}{\varphi^{k}(N)}=\lim _{n \rightarrow \infty} \frac{\varphi(n)}{n}=\frac{1}{\alpha}
$$

$$
\lim _{k \rightarrow \infty} \frac{\varphi^{k-1}(N)}{\varphi^{k}(N)}=\lim _{k \rightarrow \infty} \frac{\varphi^{k-1}(N)}{\varphi\left(\varphi^{k-1}(N)\right)}=\lim _{n \rightarrow \infty} \frac{n}{\varphi(n)}=\alpha .
$$

Hence

$$
\lim _{k \rightarrow \infty} \frac{\varphi^{k+1}(N)-\varphi^{k}(N)}{\varphi^{k}(N)-\varphi^{k-1}(N)}=\lim _{k \rightarrow \infty} \frac{\frac{\varphi^{k+1}(N)}{\varphi^{k}(N)}-1}{1-\frac{\varphi^{k-1}(N)}{\varphi^{k}(N)}}=\frac{\frac{1}{\alpha}-1}{1-\alpha}=\frac{1}{\alpha} .
$$

This proves $(\mathrm{B})$.

Theorem (The $\varphi$-ratio test). Let $\varphi: Z^{+} \rightarrow Z^{+}$be such that $\lim _{n \rightarrow \infty} \frac{n}{\varphi(n)}$ exists. Denote $\lim _{n \rightarrow \infty} \frac{n}{\varphi(n)}$ by $\alpha$ and suppose $0<\alpha<1$. Let $\left\{a_{n}\right\}$ be a positive decreasing sequence. Suppose $\lim _{n \rightarrow \infty} \frac{a_{\varphi(n)}}{a_{n}}=$ L. Then:

(i) If $L<\alpha, \sum_{n=1}^{\infty} a_{n}$ converges.

(ii) If $L>\alpha, \sum_{n=1}^{\infty} a_{n}$ diverges.

(iii) If $L=\alpha$ the test is inconclusive. 
Proof. To prove (i), suppose $L<\alpha$. Let $r$ be such that $L<r<\alpha$. Since $\lim _{n \rightarrow \infty} \frac{a_{\varphi(n)}}{a_{n}}=L$, there is an integer $N$ such that $\frac{a_{\varphi(n)}}{a_{n}}<r$ for all $n \geq N$.

Therefore $a_{\varphi(n)}<r a_{n}$ for all $n \geq N$. In particular, $a_{\varphi(N)}<r a_{N}$. We may choose $N$ large enough to satisfy the hypothesis of the lemma, so that $\left\{\varphi^{k}(N)\right\}_{k}$ is strictly increasing. Thus $\varphi^{k}(N)>\varphi^{k-1}(N) \geq N$ for all $k \in Z^{+}$. Since $\varphi^{k-1}(N) \geq N$ for all $k \in Z^{+}$, replacing $N$ with $\varphi^{k-1}(N)$ gives $a_{\varphi^{k}(N)}<r a_{\varphi^{k-1}(N)}$ for all $k \in Z^{+}$. Induction on $k$ gives $a_{\varphi^{k}(N)}<r^{k} a_{N}$ for all $k \in Z^{+}$.

Let $S_{k}=a_{\varphi^{k}(N)}+a_{\varphi^{k}(N)+1}+\ldots+a_{\varphi^{k+1}(N)-1}$. Since $\left\{a_{n}\right\}$ is decreasing and $\left\{\varphi^{k}(N)\right\}_{k}$ is increasing, the largest term in the sum $S_{k}$ is $a_{\varphi^{k}(N)}$; also, the number of terms in this sum is $\varphi^{k+1}(N)-\varphi^{k}(N)$. Hence

$$
S_{k} \leq\left[\varphi^{k+1}(N)-\varphi^{k}(N)\right] a_{\varphi^{k}(N)} .
$$

Therefore

$$
S_{k} \leq\left[\varphi^{k+1}(N)-\varphi^{k}(N] r^{k} a_{N}\right.
$$

for all $k \in Z^{+}$. Since $r<\alpha$, by the lemma,

$$
\lim _{k \rightarrow \infty} \frac{\varphi^{k+1}(N)-\varphi^{k}(N)}{\varphi^{k}(N)-\varphi^{k-1}(N)}=\frac{1}{\alpha}<\frac{1}{r} .
$$

Let $s$ be such that $\frac{1}{\alpha}<s<\frac{1}{r}$. Then there is a positive integer $M$ such that $\frac{\varphi^{k+1}(N)-\varphi^{k}(N)}{\varphi^{k}(N)-\varphi^{k-1}(N)}<$ $s$ for $k \geq M$. Therefore, for $k \geq M$, we have

$$
\begin{aligned}
& \frac{\varphi^{k+1}(N)-\varphi^{k}(N)}{\varphi^{M}(N)-\varphi^{M-1}(N)} \\
& \quad=\frac{\varphi^{k+1}(N)-\varphi^{k}(N)}{\varphi^{k}(N)-\varphi^{k-1}(N)} \cdot \frac{\varphi^{k}(N)-\varphi^{k-1}(N)}{\varphi^{k-1}(N)-\varphi^{k-2}(N)} \ldots \frac{\varphi^{M+1}(N)-\varphi^{M}(N)}{\varphi^{M}(N)-\varphi^{M-1}(N)}<s^{k+1-M} .
\end{aligned}
$$

Thus for $k \geq M$,

$$
\varphi^{k+1}(N)-\varphi^{k}(N)<\left[\varphi^{M}(N)-\varphi^{M-1}(N)\right] s^{k+1-M} .
$$

Therefore for $k \geq M$,

$$
S_{k} \leq\left[\varphi^{M}(N)-\varphi^{M-1}(N)\right] s^{k+1-M} r^{k} a_{N}=\left[\varphi^{M}(N)-\varphi^{M-1}(N)\right] s^{1-M} a_{N}(s r)^{k}
$$

and therefore

$$
\sum_{n=\varphi^{M}(N)}^{\infty} a_{n}=\sum_{k=M}^{\infty} S_{k} \leq\left[\varphi^{M}(N)-\varphi^{M-1}(N)\right] s^{1-M} a_{N} \sum_{k=M}^{\infty}(s r)^{k} .
$$

Since $s<\frac{1}{r}, \sum_{k=M}^{\infty}(s r)^{k}<\infty$, and thus the series $\sum_{n=1}^{\infty} a_{n}$ converges. This proves (i). To prove (ii), suppose $L>\alpha$. Let $r$ be such that $L>r>\alpha$. Since $\lim _{n \rightarrow \infty} \frac{a_{\varphi(n)}}{a_{n}}=L$, there is an integer $N$ such that $\frac{a_{\varphi(n)}}{a_{n}}>r$ for all $n \geq N$. Therefore $a_{\varphi(n)}>r a_{n}$ for all $n \geq N$. In 
particular, $a_{\varphi(N)}>r a_{N}$ and induction on $k$ gives $a_{\varphi^{k}(N)}>r^{k} a_{N}$ for all $k \in Z^{+}$. Let $s$ be such that $\frac{1}{r}<s<\frac{1}{\alpha}$. As in the proof of (i), by letting

$$
S_{k}=a_{\varphi^{k}(N)}+a_{\varphi^{k}(N)+1}+\ldots+a_{\varphi^{k+1}(N)-1},
$$

we can show that there is a positive integer $M$ such that for $k \geq M$,

$$
S_{k} \geq\left[\varphi^{M}(N)-\varphi^{M-1}(N)\right] s^{-M} a_{N}(s r)^{k+1} .
$$

Therefore

$$
\sum_{n=\varphi^{M}(N)}^{\infty} a_{n}=\sum_{k=M}^{\infty} S_{k} \geq\left[\varphi^{M}(N)-\varphi^{M-1}(N)\right] s^{-M} a_{N} \sum_{k=M}^{\infty}(s r)^{k+1} .
$$

Since $s>\frac{1}{r}, \sum_{k=M}^{\infty}(s r)^{k+1}$ diverges. Hence the series $\sum_{n=1}^{\infty} a_{n}$ diverges.

To prove (iii), use the examples in [1].

The following class of examples shows that for every $\varphi$ under consideration here, the $\varphi$ ratio test is stronger than the ordinary ratio test.

Example 1. Suppose that $\varphi: Z^{+} \rightarrow Z^{+}$and $\alpha$ satisfy the hypothesis of the lemma. Then the $\varphi$-ratio test works on the $p$-series, for $p \neq 1$ (although the ordinary ratio test does not).

Proof. For the $p$-series $\sum_{n=1}^{\infty} \frac{1}{n^{p}}, a_{n}=\frac{1}{n^{p}}$. Then

$$
L=\lim _{n \rightarrow \infty} \frac{a_{\varphi(n)}}{a_{n}}=\lim _{n \rightarrow \infty}\left(\frac{n}{\varphi(n)}\right)^{p}=\alpha^{p} .
$$

Since $\alpha<1, L=\alpha^{p}<\alpha$ if $p>1$ and $L=\alpha^{p}>\alpha$ if $p<1$. So, by the $\varphi$-ratio test, $\sum_{n=1}^{\infty} \frac{1}{n^{p}}$ converges if $p>1$ and diverges if $p<1$. (Note that for $p=1$, the $\varphi$-ratio test gives no information, since $L=\alpha$.)

We close this paper with another class of examples, which shows that $\varphi$ can be found to bring $\alpha=\lim _{n \rightarrow \infty} \frac{n}{\varphi(n)}$ arbitrarily close to 1 . (The $m^{\text {th }}$-ratio tests in [1] show that $\alpha=\frac{1}{m}$ can be arbitrarily close to 0 .)

Example 2. For any fixed $\varepsilon>0$, let $\varphi_{\varepsilon}: Z^{+} \rightarrow Z^{+}$be the function defined by $\varphi_{\varepsilon}(n)=$ $[[(1+\varepsilon) n]]$, the integral part of $(1+\varepsilon) n$. Then

$$
(1+\varepsilon) n-1<\varphi_{\varepsilon}(n) \leq(1+\varepsilon) n .
$$

Thus

$$
(1+\varepsilon)-\frac{1}{n}<\frac{\varphi_{\varepsilon}(n)}{n} \leq 1+\varepsilon
$$

Therefore $\lim _{n \rightarrow \infty} \frac{\varphi_{\varepsilon}(n)}{n}=1+\varepsilon$. We then have $\alpha=\lim _{n \rightarrow \infty} \frac{n}{\varphi_{\varepsilon}(n)}=\frac{1}{1+\varepsilon}<1$. Hence $\varphi_{\varepsilon}$ satisfies the hypothesis of the theorem and for small enough $\varepsilon, \alpha$ is arbitrarily close to 1 . 
There are several further areas of investigations that come to mind. First, how does the ordinary ratio test fit into all of this? It is not a special case, because if it were, we would have $\varphi(n)=n+1$, and thus $\lim _{n \rightarrow \infty} \frac{n}{\varphi(n)}=\lim _{n \rightarrow \infty} \frac{n}{n+1}=1$, not less than 1 . So are the $\varphi$-ratio tests a whole different category, rather than a generalization of the ordinary ratio test? Perhaps the ordinary ratio test is some kind of limiting case? Second, are some $\varphi$-ratio tests stronger than others? Third, are there other tests of this ilk that are not ratio tests? More specifically, are there other binary functions $F$ on $Z^{+}$(besides the quotient/ratio) for which we can say, analogous to our theorem:

For all positive decreasing sequences $\left\{a_{n}\right\}$, we have:

$$
\lim _{n \rightarrow \infty} F\left(a_{\varphi(n)}, a_{n}\right)<\lim _{n \rightarrow \infty} F(n, \varphi(n))
$$

implies that $\sum_{n=1}^{\infty} a_{n}$ is convergent, and

$$
\lim _{n \rightarrow \infty} F\left(a_{\varphi(n)}, a_{n}\right)>\lim _{n \rightarrow \infty} F(n, \varphi(n))
$$

implies that $\sum_{n=1}^{\infty} a_{n}$ is divergent?

\section{References}

[1] Ali, S.: The $m^{\text {th }}$ Ratio Tests. Amer. Math. Monthly 115 (2008) 6, 514-524.

Sayel Ali

Mathematics Department

Minnesota State University Moorhead

Moorhead, Minnesota, USA

e-mail: alis@mnstate.edu

Marion Deutsche Cohen

Department of Computer Science and Mathematics

Arcadia University, PA, USA

e-mail: cohenmaarcadia.edu 